\title{
ArcheoSciences
}

Revue d'archéométrie

38 | 2014

Varia

\section{Découvertes de résidus de préparations alimentaires dans quatre sites gaulois du Centre}

Discoveries of culinary preparations residues in four Gallic Center sites

Bénédicte Pradat

\section{CpenEdition}

1 Journals

Édition électronique

URL : https://journals.openedition.org/archeosciences/4184

DOI : 10.4000/archeosciences.4184

ISBN : 978-2-7535-3691-3

ISSN : 2104-3728

Éditeur

Presses universitaires de Rennes

Édition imprimée

Date de publication : 30 novembre 2014

Pagination : $97-108$

ISBN : 978-2-7535-3689-0

ISSN : $1960-1360$

Référence électronique

Bénédicte Pradat, « Découvertes de résidus de préparations alimentaires dans quatre sites gaulois du Centre ", ArcheoSciences [En ligne], 38 | 2014, mis en ligne le 30 novembre 2016, consulté le 31 janvier 2022. URL : http://journals.openedition.org/archeosciences/4184; DOI : https://doi.org/10.4000/ archeosciences. 4184 


\title{
Découvertes de résidus de préparations alimentaires dans quatre sites gaulois du Centre
}

\author{
Discoveries of Culinary Preparations Residues in four Gallic Center Sites
}

\author{
Bénédicte PradaT ${ }^{\mathrm{a}}$
}

\begin{abstract}
Résumé : La découverte de quatre caramels alimentaires en région Centre sur des sites de La Tène a incité à caractériser plus précisément les résidus de préparations alimentaires détectés parmi les vestiges archéologiques. Divers procédés de préparatifs culinaires ont été répertoriés pour lesquels sont détaillés les différentes formes sous lesquels un aliment végétal sec peut être utilisé ainsi que le type de préparation obtenue. Grâce à une observation minutieuse des résidus archéologiques et en se référant aux critères précédemment établis, des hypothèses sont émises sur les types de préparations qui ont été élaborés à l'âge du Fer.

Abstract: The detection of four caramels in the context of La Tene sites in the Center of France has led to further investigations in view to specify food residues identified among the archaeological finds. Various methods of culinary preparation have been listed. Different use of dry plant aliment and the type of preparation obtained are detailed. Through careful observation of archaeological residues and by reference to previously established criteria, assumptions are made about the types of preparations that have been developed during the Iron Age.
\end{abstract}

Mots clés : La Tène, région Centre, préparations alimentaires.

Keywords: Iron age La Tene, central region of France, culinary preparations.

Suite à la multiplication des études carpologiques et à un travail collectif mené sur les habitats ruraux (Zech-Matterne et al., 2009), et ce malgré quelques lacunes géographiques qui persistent, le panel des espèces végétales consommées en Gaule au cours de l'âge du Fer commence à être bien connu. Mais, si les plantes consommées sont bien répertoriées, les données textuelles concernant leur accommodation font défaut. Aucun livre de recettes n'est en effet disponible pour cette période, nous privant ainsi d'indications sur la façon de cuisiner ou les usages culinaires. Toutefois, des informations sur ce sujet peuvent nous parvenir par d'autres moyens. La préservation de résidus alimentaires en est un car, s'ils ne livrent pas de recettes, leur observation fournit néanmoins des indices sur les types de préparations qui ont été effectués.
Dans la région Centre, des « caramels alimentaires » étaient préservés dans quatre sites datés de La Tène : à Sublaines et Saint-Georges (Indre-et-Loire, A 85), à Corbeilles (Loiret, A 19) et à Saran (Loiret). Suite à une observation minutieuse de ces résidus, l'identification de ces macrorestes est tentée à partir des sources écrites et des pratiques culinaires encore en cours.

\section{LES CONTEXTES DE DÉCOUVERTES}

Pour l'ensemble des sites, les prélèvements ayant fait l'objet d'une étude carpologique ont été tamisés par les équipes de fouille sur des cribles de mailles 2 et $0,5 \mathrm{~mm}$. L'extraction

a Inrap, UMR 7209 CNRS-Muséum d'Histoire naturelle - 148 avenue Maginot, 37100 ToURS. (benedicte.pradat@inrap.fr) 
des semences a ensuite été effectuée sous loupe binoculaire par la carpologue.

\section{Le site de Sublaines " Le Grand Ormeau » (Indre-et-Loire) $^{1}$}

Entre le Néolithique et le Bronze final, diverses occupations se succèdent puis, après un hiatus chronologique, un établissement rural est installé à La Tène ancienne. Celui-ci est constitué d'aménagements liés à un complexe funéraire à l'est et d'un habitat caractérisé par des silos, des palissades et des bâtiments à l'ouest. Trois phases comprises entre La Tène $A$ et $C$ ont pu être distinguées (Frénée $e t$ al., 2008).

\section{Les résultats carpologiques du site}

L'étude carpologique du site de Sublaines a porté sur une quarantaine de comblements de silos datés de la fin du Hallstatt D3/La Tène A1 ( $v^{e} s$. avant notre ère), de la Tène A2-B1 (seconde moitié IV $v^{e}$-première moitié $I V^{e} s$. av. notre ère) et $\mathrm{B} 2-\mathrm{C} 1$ (seconde moitié $\mathrm{IV}^{\mathrm{e}}$-première moitié $\mathrm{III}^{\mathrm{e}} \mathrm{s}$. av. notre ère). Au total, ce sont plus de 7000 restes qui ont été isolés. Ils correspondent à 18 espèces domestiques et à plus de 70 taxons sauvages. Ce site présente donc une richesse en restes carpologiques, tant par le nombre de restes attestés que par sa variété de taxons.

La grande majorité des restes attestés est conservée sous forme carbonisée (tableau 1). Les céréales ont pour la plupart un aspect assez alvéolaire avec un épiderme mal conservé. Leur apparence est nettement déformée. Cette conservation assez désastreuse ne permet pas d'identifier à l'espèce certaines céréales (Triticum sp.) et, bien souvent, les fragments n'ont pu être reconnus que comme appartenant au groupe "céréales" (Cerealia) sans qu'une espèce particulière ne puisse être privilégiée.

Les furcas d'amidonnier/engrain (fourches sur lesquelles reposent les grains) sont généralement fragmentées et très altérées, ce qui rend alors impossible la précision de l'appartenance à l'une ou l'autre des espèces. Quelques restes, moins d'une trentaine, sont préservés par minéralisation.

L'assemblage carpologique est dominé par deux plantes cultivées. La première, et la plus importante, est le blé vêtu de type amidonnier ou engrain (Triticum dicoccum/monococcum) qui est attesté par des grains et, pour l'essentiel, par des furcas. L'orge vêtue (Hordeum vulgare) représente le second taxon dominant. Les plantes mineures comprennent dix espèces domestiques - les deux millets (Panicum miliaceum et Setaria italica), le blé type froment (Triticum aestivum l.s.), l'épeautre (Triticum spelta), la lentille (Lens

1. Fouilles sous la direction d'Eric Frénée, Inrap. culinaris), le pois (Pisum sativum), la féverole (Vicia faba), l'ers (Vicia ervilia) et la vesce (Vicia sativa), des fruits sauvages cueillis - noisette (Corylus avellana), pomme (Malus sp.), prunelle (Prunus spinosa), glands de chêne (Quercus sp.), mûre ronce (Rubus fruticosus) et un pépin de vigne (Vitis $s p$.) mal conservé mais qui, d'après sa forme trapue, semble provenir de l'espèce sauvage, et une plante condimentaire, la moutarde noire (Brassica nigra). Par ailleurs, un nombre très important d'adventices des cultures et autres espèces sauvages est conservé sur l'ensemble du site.

\section{De la matière organique (figure 1)}

D'autre part, de la matière organique carbonisée est également préservée dans de nombreuses structures. La plupart des fragments, de petite taille, à la texture grossière et ne renfermant aucune semence, ne seront pas commentés car leur dimensions ne permettent pas une étude approfondie. Dans le comblement du silo 2032 (US 4), daté de La Tène A2-B1, des traces de tiges ont été observées sur certains des 185 fragments, dont les plus grands font $8 \mathrm{~mm}$ de long. Est-ce un aliment (fruit, fromage, viande...) mis à sécher sur un clayonnage qui aurait pris feu?

Dans ce même silo (US 22), d'autres résidus de matière organique, très nombreux, retiennent l'attention : ils présentent une texture assez grossière, constituée de parties « filandreuses » agglomérées les unes aux autres. Par ailleurs, des glands sont attestés dans cette même couche : 9 cotylédons et environ 700 fragments. La plupart d'entre eux sont recouverts d'une matière organique telle une pâte collante les enrobant.

En observant mieux cette dernière, on discerne, dans certains de ces morceaux, des fragments de glands dont l'aspect d'origine est quelque peu modifié. Citons pour exemple un gland dont une extrémité est parfaitement reconnaissable tandis que l'autre est déformée, comme " mâchée ".

\section{Le site d'Epeigné-les-Bois "Les Allets »/ Saint-Georges-sur-Cher "Le Marchais rond » (Indre-et-Loire) $^{2}$}

Le site, occupé de façon discontinue du Paléolithique moyen jusqu'au Moyen Âge, est caractérisé pour l'essentiel par un vaste établissement rural à La Tène moyenne (III $\mathrm{II}^{\mathrm{e}} \mathrm{s}$.), ainsi qu'à La Tène finale-début de l'époque augustéenne (40 av.-10 apr. J.-C.), date de son abandon.

L'établissement se compose de deux enclos constitués de fossés larges et profonds, de treize bâtiments, de fosses dépotoirs et de foyers. La présence de pratiques cultuelles,

2. Fouilles sous la direction d'Agnès Couderc, Inrap. 


\begin{tabular}{|c|c|c|c|c|c|c|c|c|}
\hline & & & \multirow{2}{*}{\multicolumn{2}{|c|}{$\begin{array}{c}\text { fin Ha D3- La Tène A } \\
5 \text { prélèvements }\end{array}$}} & \multirow{2}{*}{\multicolumn{2}{|c|}{$\begin{array}{l}\text { La Tène A2-B1 } \\
10 \text { prélèvements }\end{array}$}} & \multicolumn{2}{|c|}{ La Tène B2-C1 } \\
\hline & & & & & & & \multicolumn{2}{|c|}{15 prélèvements } \\
\hline & & & NR total & fréquence & NR total & fréquence & NR total & fréquence \\
\hline \multicolumn{9}{|l|}{ CÉRÉALES grains } \\
\hline Hordeum vulgare & Orge polystique vêtue & semence & 76 & 4 & 51 & 9 & 26 & 7 \\
\hline Panicum miliaceum & Millet commun & semence & 5 & 1 & 13 & 5 & 24 & 11 \\
\hline Setaria italica & Millet des oiseaux & semence & 6 & 2 & & & & \\
\hline Triticum aestivum l.s. & Blé type froment & semence & 4 & 1 & 24 & 6 & 9 & 5 \\
\hline Triticum dicoccum & Amidonnier & semence & 64 & 3 & 23 & 8 & 10 & 5 \\
\hline Triticum monococcum & Engrain & semence & 4 & 1 & 3 & 2 & 3 & 2 \\
\hline Triticum cf. spelta & Epeautre & semence & & & 2 & 1 & & \\
\hline Triticum sp. & Blé & semence & 11 & 1 & 15 & 7 & 27 & 8 \\
\hline Cerealia & Céréales & semence & 56 & 3 & 105 & 8 & 96 & 15 \\
\hline \multicolumn{3}{|c|}{ TOTAL céréales grains } & 226 & & 236 & & 195 & \\
\hline \multicolumn{9}{|l|}{ CÉRÉALES vannes } \\
\hline Avena sp. & Avoine & fgt de barbe & 24 & 3 & 27 & 5 & 7 & 5 \\
\hline Avena fatua & Folle-avoine & $\begin{array}{l}\text { base de } \\
\text { lemme }\end{array}$ & 1 & 1 & 1 & 1 & & \\
\hline Hordeum vulgare & Orge polystique vêtue & fgt de rachis & 3 & 2 & 2 & 2 & 1 & 1 \\
\hline Triticum dicoccum & Amidonnier & furca & 16 & 2 & 23 & 4 & 12 & 8 \\
\hline Triticum monococcum & Engrain & furca & 7 & 2 & 29 & 5 & 29 & 8 \\
\hline $\begin{array}{l}\text { Triticum dicoccum/monococ- } \\
\text { cum }\end{array}$ & Amidonnier/Engrain & furca & 151 & 2 & 299 & 10 & 346 & 15 \\
\hline Triticum cf. spelta & Epeautre & furca & 11 & 1 & 18 & 6 & 7 & 6 \\
\hline Triticum sp. & Blé & fgt de rachis & 10 & 1 & 4 & 3 & 5 & 2 \\
\hline Cerealia & Céréales & fgt de tige & 8 & 1 & & & & \\
\hline \multicolumn{3}{|c|}{ TOTAL céréales vannes } & 231 & & 403 & & 407 & \\
\hline \multicolumn{9}{|l|}{ LÉGUMINEUSES } \\
\hline Lens culinaris & Lentille & semence & & & 14 & 4 & 29 & 5 \\
\hline Pisum sativum & Pois & semence & & & 4 & 3 & & \\
\hline Vicia ervilia & Ers & semence & 3 & 1 & 24 & 7 & 53 & 7 \\
\hline Vicia faba & Féverole & semence & & & 2 & 2 & & \\
\hline Vicia sativa & Vesce & semence & 1 & 1 & & & 2 & 2 \\
\hline cf. Vicia sativa & Vesce & semence & & & 3 & 1 & & \\
\hline Vicia/Lens/Pisum & Vesce/Lentille/Pois & semence & 10 & 4 & 15 & 6 & 22 & 6 \\
\hline \multicolumn{3}{|c|}{ TOTAL légumineuses } & 14 & & 62 & & 106 & \\
\hline \multicolumn{9}{|l|}{ FRUITS } \\
\hline Corylus avellana & Noisetier & fgt de coque & 1 & 1 & 3 & 3 & 6 & 6 \\
\hline Malus sp. & Pommier & fgt de pépin & & & 1 & 1 & & \\
\hline Prunus spinosa & Prunellier & fgt de noyau & 1 & 1 & 2 & 2 & & \\
\hline Quercus sp. & Chêne & gland & 1 & 1 & 5 & 1 & 14 & 6 \\
\hline Quercus sp. & Chêne & germe & & & 3 & 2 & & \\
\hline Quercus sp. & Chêne & $\begin{array}{c}\text { base tégu- } \\
\text { ment }\end{array}$ & & & & & 1 & 1 \\
\hline Rubus fruticosus & Mûrier ronce & semence & & & 1 & 1 & & \\
\hline Vitis sp. & Vigne sauvage & pépin & 1 & 1 & & & & \\
\hline \multicolumn{3}{|c|}{$\begin{array}{l}\text { TOTAL fruits } \\
\end{array}$} & 4 & & 15 & & 21 & \\
\hline \multicolumn{9}{|c|}{\begin{tabular}{l|l} 
OLÉAGINEUX/TEXTILE & \\
\end{tabular}} \\
\hline Brassica nigra & Moutarde noire & semence & 3 & 1 & & & 6 & 5 \\
\hline \multicolumn{3}{|c|}{ TOTAL oléagineux/textile } & 3 & & & & 6 & \\
\hline \multicolumn{3}{|c|}{ TOTAL Plantes cultivées } & 478 & & 716 & & 735 & \\
\hline
\end{tabular}

Tableau 1 : restes carpologiques du site de Sublaines (NR : nombre de restes, calculés en nombre minimum d'individus; fgt : fragment). Table 1: carpological remains of Sublaines site (NR: number of remains, calculated minimum number of individuals; fgt: fragment). 




b

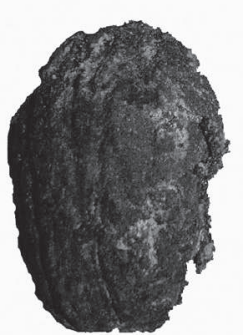

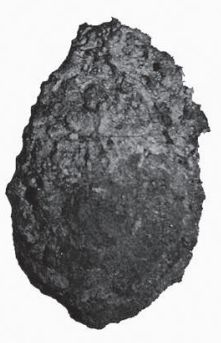

C
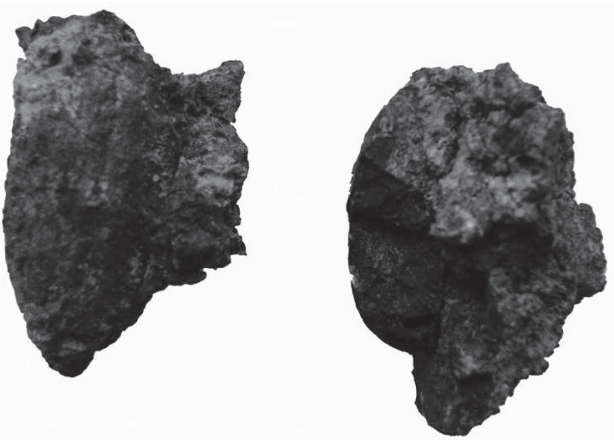

$\mathrm{d}$

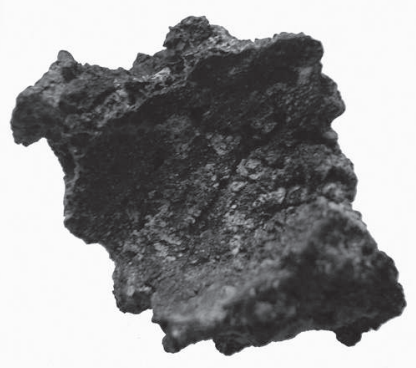

Figure 1 : Les résidus de Sublaines. a- quelques cotylédons de glands (Quercus) et de la matière organique; b- cotylédons; c- cotylédons/ matière organique; d- empreinte de cotylédon dans de la matière organique (photo F. Di Napoli).

Figure 1: Residues Sublaines. a-some cotyledons of glands (Quercus) and organic matter; b-cotyledons; c-cotyledons/organic matter; $d$ - cotyledon footprint in organic matter (photo F. Di Napoli).

mais surtout la superficie, les dimensions et l'organisation des structures confèrent à cet établissement un statut relativement élevé dans la hiérarchie des fermes rurales gauloises (Couderc et al., 2006).

Le résidu carpologique qui nous concerne provient d'un des fossés d'enclos dont le comblement est daté de La Tène D1 (150-80 avant notre ère) par la céramique ${ }^{3}$.

\section{Les résultats carpologiques du site}

Parmi les vingt-sept échantillons qui nous ont été confiés, seuls quatre prélèvements contenaient des restes carpologiques qui, par ailleurs, sont peu nombreux puisque seuls une soixantaine de restes au total ont été isolés : ils correspondent à trois plantes cultivées, une plante sauvage et de la matière organique.

Tous les restes attestés sont conservés sous forme carbonisée. Les céréales ont un état de conservation moyen. Cependant, les fragments présentent une altération plus marquée qui n'a pas permis de les attribuer à une espèce (Cerealia).

3. Étude céramique réalisée par F. Di Napoli, Inrap.
Les restes découverts sur le site (tableau 2) appartiennent essentiellement à des céréales. Les espèces attestées sont surtout de l'orge vêtue (Hordeum vulgare) qui est représentée par des semences mais aussi par une base de lemme (base de l'enveloppe qui entoure les grains). Un grain de blé type froment (Triticum aestivum l.s.) et deux d'amidonnier (Triticum dicoccum) sont également repérés.

Une semence d'avoine (Avena sp.) est aussi conservée. En l'absence de base de lemme, on ne peut savoir s'il s'agit d'une espèce sauvage ou cultivée. Exceptée cette plante, aucune espèce sauvage n’a été mise en évidence.

\section{Un agglomérat carbonisé (figure 2)}

De la matière organique carbonisée a pu être observée parmi laquelle un fragment présente des alvéoles de tailles disparates et non organisées (F26). Aucun élément végétal n'y ayant par ailleurs été décelé, il est impossible de savoir à quoi correspond cette matière.

Deux blocs compacts de matière organique provenant du fossé F64 sont en revanche plus intéressants. Ces agglomérats ont une taille respective de $3,5 \times 3 \mathrm{~cm}$ et $5,5 \times 4 \mathrm{~cm}$ sur environ $1 \mathrm{~cm}$ d'épaisseur. Leur forme est légèrement incur- 


\begin{tabular}{|c|c|c|c|}
\hline Nom scientifique & nom français & type de reste & nombre total de restes \\
\hline \multicolumn{4}{|l|}{ CÉRÉALES grains } \\
\hline Hordeum vulgare & Orge polystique vêtue & semence & minimum 27 \\
\hline Hordeum vulgare & Orge polystique vêtue & fgt de semence & 4 \\
\hline Triticum aestivum l.s. & Blé type froment & semence & 1 \\
\hline Triticum dicoccum & Amidonnier & semence & 2 \\
\hline Cerealia & Céréales & fgt de semence & 20 \\
\hline \multicolumn{4}{|l|}{ CÉRÉALES vannes } \\
\hline Hordeum vulgare & Orge polystique vêtue & base de lemme & 2 \\
\hline \multicolumn{4}{|l|}{ PLANTES SAUVAGES } \\
\hline Avena sp. & Avoine & semence & 1 \\
\hline \multicolumn{4}{|l|}{ AUTRES } \\
\hline Matière organique & & fgt & ** \\
\hline Matière organique indéterminée & & fgt & 1 \\
\hline
\end{tabular}

Tableau 2 : restes carpologiques du site d'Epeigné/Saint Georges, en nombre minimum d'individus (fgt : fragment).

Table 2: carpological remains of Epeigné/Saint Georges site, in minimum number of individuals (fgt : fragment).

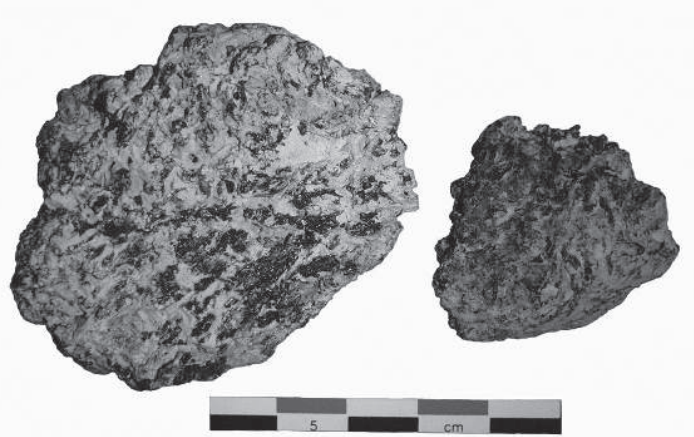

b

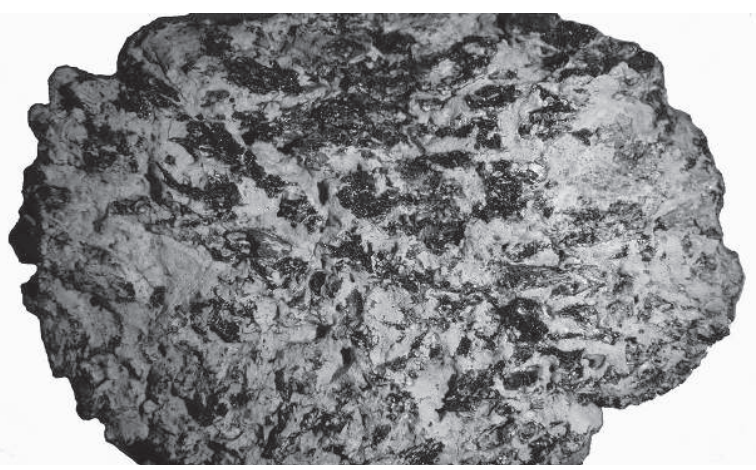

Figure 2 : Les amas d'orge de Saint-Georges. a- les blocs incurvés; b- détail d'un bloc (un grain d'orge est visible) (photo F. Di Napoli). Figure 2: Saint-Georges's clusters barley. a-curved blocks; b-detail of a block (a barley grain is visible) (photo F. Di Napoli).

vée sur une des surfaces et présente des cassures sur l'autre. La texture de ces amas est assez grossière et non régulière. Plusieurs semences de céréales, correspondant à de l'orge, y sont emprisonnées. Ces grains sont cependant assez épars et ne constituent pas l'essentiel des blocs. Quelques uns se sont détachés au cours des diverses manipulations (tamisage, observation sous loupe binoculaire...). Ils sont d'assez petite taille puisque leur longueur est comprise entre 4,1 et $4,9 \mathrm{~mm}$ et leur largeur entre 1,8 et $2,2 \mathrm{~mm}$ avec une moyenne de $4,5 \times 2 \mathrm{~mm}(\mathrm{NR}=6)$. En comparaison avec d'autres sites laténiens régionaux, ils sont légèrement plus petits que des grains d'orge issus d'une récolte brûlée à Lazenay (Bourges, Cher) et nettement inférieurs à ceux trouvés au Grand Jaunet (Liniez, Indre) qui mesurent en moyenne respectivement $4,73 \times 2,44$ et 5,6 x 2,9 mm (NR = 100) (Pradat, 2001). La conservation des caryopses est assez bonne. Certains possèdent sur leur surface quelques lambeaux de glumes (enveloppe qui entoure le grain) et on observe au sein des blocs des fragments de glumes et même un grain d'orge encore vêtu.

\section{Le site de Corbeilles-en-Gâtinais "Franchambault " (Loiret) ${ }^{4}$}

\section{Le contexte archéologique}

Hormis quelques tessons de céramique du Bronze ancien en position résiduelle, c'est à partir du Hallstatt final et La Tène ancienne que le site est occupé. Il se caractérise par des bâtiments sur poteaux (dont des greniers), un fond de cabane, des silos, des puits et des fosses. Après un abandon, le secteur est de nouveau occupé à partir de la période augustéenne (Poitevin et al., 2007).

4. Fouilles sous la direction de Grégory Poitevin, Inrap. 


\section{Un caramel alimentaire (figure 3)}

Au cours de l'étude céramologique, F. Di Napoli a remarqué un résidu carbonisé conservé dans le fond d'un vase quasi complet provenant d'un puits (F257 US 2101) dont le comblement est daté du Hallstatt final/La Tène A (vI$v^{e} s$. avant notre ère). Ce vase présente des traces nettes de découpe au niveau de la panse montrant la volonté d'une réutilisation du récipient pour un usage différent (Di Napoli in Poitevin et al., 2007).

La couche carbonisée préservée au fond du vase de Corbeilles est d'environ $1 \mathrm{~mm}$ d'épaisseur. Son observation sous loupe binoculaire montre une texture assez fine mais irrégulière : de nombreuses alvéoles (sortes de petites bulles) sont visibles. En de nombreux endroits elle a un aspect brillant de type vitreux. Quelques rares éléments fibreux
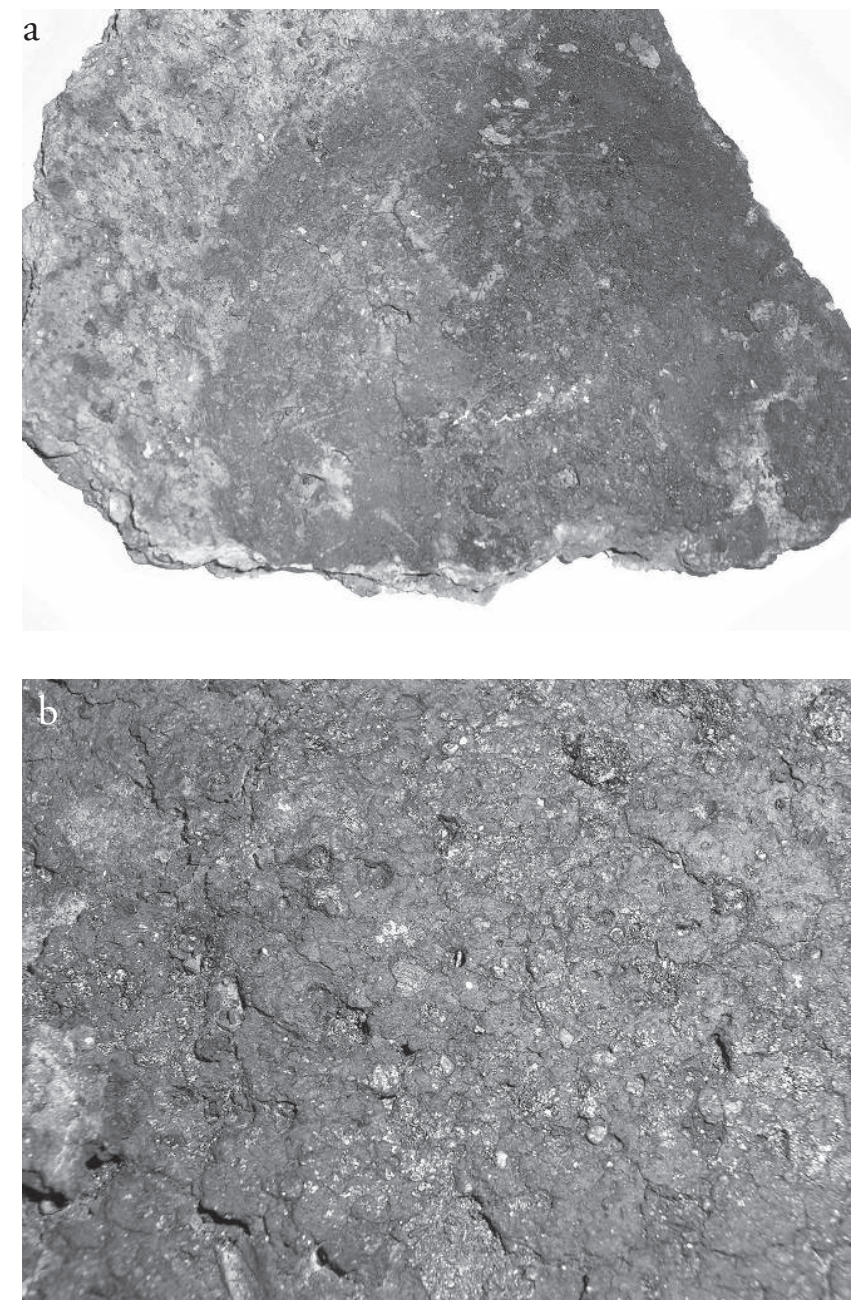

Figure 3 : (Voir planche couleur) Le caramel de Corbeilles. a- le contenant b- détail (photo F. Di Napoli).

Figure 3: (See colour plate) Corbeilles's caramel. a-container; $b$-detail (photo F. Di Napoli). sont conservés. Quatre d'entre eux ont pu être isolés, tous inférieurs à $1 \mathrm{~mm}$ de long. Ils présentent un alignement cellulaire typique des glumes de millet commun (Panicum miliaceum).

Cette couche se détache par petites plaques. Lorsque l'on soulève celles-ci, la pâte de la céramique se desquame. Par ailleurs, quelques cristaux de dégraissant sont mêlés à la couche carbonisée. Ce résidu a donc bien brûlé dans le vase.

\section{Le site de Saran "ZAC du Champ Rouge " (Loiret) $^{5}$}

\section{Le contexte archéologique}

Des établissements ruraux caractérisés par des enclos, des tranchées de fondation et des constructions sur poteaux ont été mis au jour. Ils sont installés dès le milieu du $\mathrm{II}^{\mathrm{e}} \mathrm{s}$. avant notre ère et se poursuivent sans discontinuité jusqu'au $\mathrm{I}^{\mathrm{er}} \mathrm{s}$. de notre ère (Lusson et al., 2009).

\section{Un caramel alimentaire (figure 4)}

Parmi le mobilier céramique étudié par J.-P. Gay, un fragment de fond de dolium présente un caramel alimentaire. Il provient d'un trou de poteau (F3768 US 37170) rattaché à un bâtiment, d'habitat très vraisemblablement, dont la datation est de la fin de La Tène C2/La Tène D1. À l'observation sous loupe binoculaire, il apparaît que la couche brûlée au fond du vase est très mince (moins d'un millimètre d'épaisseur). La texture est régulière, très fine et aucune semence ou autre élément végétal n'est visible.

Ces quatre sites ont donc livré des résidus alimentaires variés. Afin de comprendre à quoi ils correspondent, il convient dans un premier temps de définis quels sont les divers types de préparations qu'il est possible de rencontrer.

\section{DES PRÉPARATIONS ALIMENTAIRES?}

Le champ de la confection culinaire peut être vaste : de l'épluchage de légumes ou encore du dénoyautage ou épépinage des fruits à la cuisson élaborée des aliments. Il faut donc tout d'abord s'interroger sur le type d'action à considérer comme étant une préparation alimentaire.

Dans le présent article, est considérée comme préparation alimentaire, tout acte visant à transformer les végétaux, de façon plus ou moins élaborée, afin de les rendre consommables. De ce fait, les préparations agricoles (battages, vannages...) ne sont pas prises en considération car, si elles sont indispensables dans les phases de traitement destinées à

5. Fouilles sous la direction de Dorothée Lusson, Inrap. 


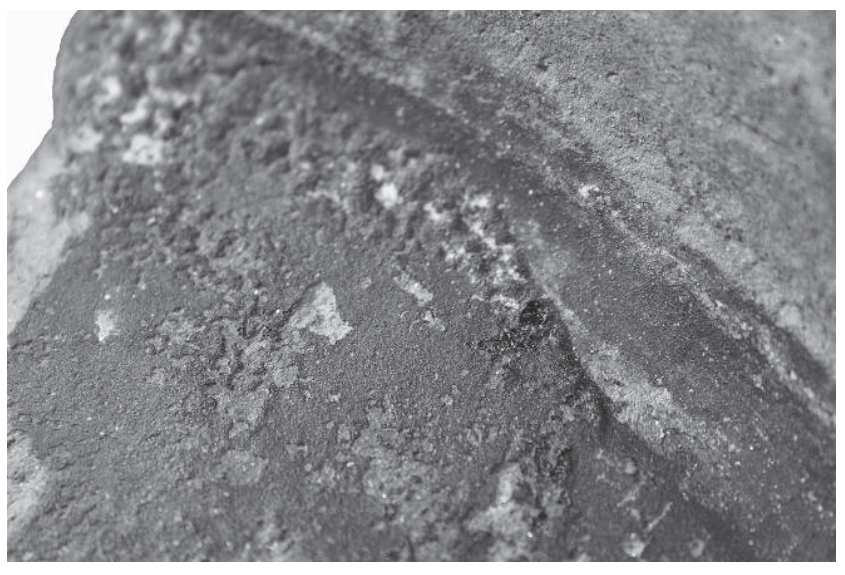

Figure 4: (Voir planche couleur) Le caramel de Saran (photo J.-P. Gay). Figure 4: (See colour plate) Saran's caramel (photo J.-P. Gay).

rendre propre à la consommation les céréales ou les légumineuses, elles ne permettent pas d'obtenir un produit directement ingérable en l'état mais correspondent à une phase de nettoyage des grains. De même, les fruits entiers carbonisés ne sont pas pris en compte dans ce travail car, s'ils sont mis à sécher et donc transformés, le processus relève alors d'un moyen de conservation et non d'une préparation.

Par ailleurs, il ne sera pas traité des parties végétales qui ne laissent pas de traces carpologiques reconnaissables (feuilles, racines...).

Les préparations alimentaires à base de végétaux peuvent être élaborées selon plusieurs procédés, tant dans la façon d'accommoder les végétaux eux-mêmes que dans leur cuisson.

Les produits végétaux « secs ", grains ou fruits secs, sont utilisés dans l'alimentation sous différents aspects.

Tout d'abord, leur usage sous forme entière est largement répandu. On pourrait citer, comme exemple parmi tant d'autres, l'emploi de céréales mises à tremper puis cuites entières dans des soupes traditionnelles - par exemple la soupe d'épeautre des Alpes du sud (Escallier \& Musset, 1991).

Les grains ou fruits secs peuvent aussi être concassés plus ou moins finement. En procédant à un broyage grossier de grains de diverses céréales décortiqués, on obtient un gruau, appellation dérivée vers 1170 du mot plus ancien "gru ". Le passage dans des tamis à mailles diverses permet d'obtenir des gruaux de divers diamètres. La semoule, mot qui apparaît au tout début du XVI ${ }^{\mathrm{e}}$ s., provient également d'un concassage des grains de céréales débarrassés de leur enveloppe (Horde, 2004). Elle est plus ou moins fine mais se différencie du gruau par la taille des résidus de broyage : le gruau est plus grossier, la semoule plus fine.
Enfin, les éléments végétaux « secs " peuvent être réduits en poudre. Le résultat est alors une farine dont l'origine n'est pas strictement céréalière mais peut aussi être obtenue à partir de légumineuses (farine de pois-chiches, de feves...), de fruits secs (glands, noisettes...) ou encore de semences de plantes sauvages (chénopodes par exemple - Jacquat, 1989).

Le gruau, la semoule et la farine ne sont pas des préparations alimentaires au sens où nous l'avons défini plus haut mais peuvent constituer un préalable à la préparation culinaire.

Par ailleurs, les types de cuisson vont influer sur le résultat final de la préparation. Ils peuvent être distingués en trois groupes.

\section{Les préparations sans cuisson}

Les aliments végétaux peuvent subir des transformations par le biais de fermentation. La lacto-fermentation permet, entre autres, d'augmenter les apports en vitamines et acides aminés des végétaux (Labbé, 1997). C'est le cas bien connu de la choucroute qui provient d'une fermentation de feuilles de choux. Ce procédé est particulièrement difficile à mettre en évidence par la carpologie car les éléments végétaux utilisés (feuilles, racines) sont râpés ou coupés fins et ne pourraient donc être discernables au sein d'amas de végétaux tels qu'on en trouve parfois en contextes humides.

La saumure, autre méthode de modification du végétal sans cuisson au feu, n'est pas prise en compte dans cet article car c'est avant tout le procédé de conservation qui est recherché dans son utilisation plus que celui de la transformation en vue d'une consommation.

Enfin, la fermentation de fruits ou de céréales peut aboutir à des boissons alcoolisées. Par exemple, la bière est réalisée à partir d'un mélange d'eau, de malt (céréales germées et torréfiées) et de plantes aromatiques, tel le houblon, que l'on met à fermenter.

\section{Les préparations avec cuisson}

\section{Cuisson sans liquide}

Une cuisson "à sec " permet de rôtir, griller, torréfier. Le grillage donne aux végétaux un aspect croustillant et plus savoureux. La torréfaction, qui consiste à exposer des graines à une température élevée, entraîne la transformation de l'amidon qu'elles contiennent en sucre et les rend plus digestes et meilleures (Jacquat, 1989). Actuellement, l'orge grillée est consommée comme friandise en Ethiopie ${ }^{6}$. La marmelade est aussi le résultat d'une cuisson sans eau

6. Informations orales d'A.-M. Jouquand. 
puisque les fruits sont cuits avec du sucre, suffisamment longtemps pour qu'ils perdent leur forme et que la peau et le reste soient mêlés ensemble et forment un ensemble assez consistant. Par extension, il se dit parfois d'une chose trop cuite et presque en bouillie.

\section{Cuisson avec liquide}

Les cuissons à l'eau transforment les restes végétaux de façon plus prononcée puisque c'est leur texture même qui va être modifiée. Un élément sec et dur sera ainsi amolli.

Dans le cas de cuisson par décoction, il faut distinguer les soupes, les bouillies et les purées.

D'après les définitions relevées dans divers dictionnaires ${ }^{7}$, la soupe est un met liquide épaissi avec du pain ou autres ingrédients (légumes, poissons, charcuteries, pâtes...) non passés, à la différence du potage qui ne contient pas de morceaux.

La purée est obtenue en foulant et en passant au tamis des aliments cuits additionnés le plus souvent d'un liquide pour les rendre plus fluides. Les purées sont de consistance épaisse.

La bouillie, mot ancien du vocabulaire français, relevé au $\mathrm{XII}^{\mathrm{e}}$ s., dérivé du verbe bouillir, est un aliment constitué de farine, de lait, d'eau ou autre liquide, à consistance pâteuse plus ou moins épaisse. On appelle également sous ce terme un aliment pâteux composé de nourritures bouillies et écrasées.

Le terme «bouillie » traduit des réalités diverses comme le montrent les descriptions de A. Maurizio (1932) : les grains peuvent être cuisinés entiers, concassés, décortiqués ou non, sous forme de farine. Les bouillies peuvent en outre mêler plusieurs de ces ingrédients, auxquels est ajouté un liquide (eau, lait), puis longuement cuits pour obtenir un aspect mou.

On peut par ailleurs ajouter à ces bases céréalières des fruits ou des légumineuses. Le terme de bouillie peut donc recouvrir un large spectre de préparations. Le résultat attendu est néanmoins le même : une mixture de type pâteuse.

Ainsi, selon le type d'ingrédient de base utilisé, les soupes et les bouillies se distinguent parfois par la quantité d'eau qu'elles contiennent et, en conséquence, par la consistance obtenue après cuisson. Elles peuvent ainsi être élaborées à partir de grains entiers de céréales cuits en décoction dans un liquide, de grains concassés ou encore de farine. Pour une bouillie, le temps de cuisson sera plus long afin de lui conférer un aspect pâteux.

7. Définitions établies d'après Horde 2004, Le Grand Larousse encyclopédique, Larousse gastronomique, Le Petit Robert.
Si l'on combine les types de cuisson à la forme initiale des aliments utilisés, les informations précédentes peuvent être résumées sous forme de tableaux comme suit :

\begin{tabular}{|c|c|c|}
\hline $\begin{array}{l}\text { Forme des végétaux } \\
\text { utilisés couplée au } \\
\text { type de cuisson }\end{array}$ & $\begin{array}{l}\text { Type de texture } \\
\text { obtenue }\end{array}$ & $\begin{array}{c}\text { Terminologie de la } \\
\text { préparation }\end{array}$ \\
\hline $\begin{array}{l}\text { Grains ou fruits secs } \\
\text { entiers grillés à sec }\end{array}$ & $\begin{array}{c}\text { Grains ou fruits secs } \\
\text { torréfiés }\end{array}$ & "grillage" \\
\hline $\begin{array}{c}\text { Grains ou fruits secs } \\
\text { entiers } \\
+ \text { liquide }\end{array}$ & $\begin{array}{l}\text { Pâte avec des grains } \\
\text { ou fruits entiers ou } \\
\text { fragmentés }\end{array}$ & $\begin{array}{l}\text { soupe } \\
\text { bouillie } \\
\text { purée }\end{array}$ \\
\hline Gruau + liquide & $\begin{array}{l}\text { Pâte avec des frag- } \\
\text { ments de végétaux }\end{array}$ & $\begin{array}{l}\text { soupe } \\
\text { bouillie }\end{array}$ \\
\hline Farine + liquide & $\begin{array}{l}\text { Pâte régulière, com- } \\
\text { pacte, solide }\end{array}$ & $\begin{array}{l}\text { pain, galette } \\
\text { bouillie }\end{array}$ \\
\hline
\end{tabular}

Tableau 3 : type de préparation obtenue en fonction du mode de cuisson et de la forme des végétaux utilisée.

Table 3: type of preparation obtained according to the method of cooking and the shape of the plant used.

\section{INTERPRÉTATION DES RÉSIDUS ALIMENTAIRES ARCHÉOLOGIQUES}

Les sites décrits ont la particularité d'avoir livré des résidus de matière organique carbonisée qui sont les témoins d'activités culinaires. Grâce à l'observation de ces « agglomérats ", nous avons pu émettre des hypothèses quant aux types de préparations qui ont été effectuées.

\section{Une " pâte " de glands à Sublaines}

D'après les observations faites sur les résidus, la matière organique et les cotylédons de glands apparaissent mêlés, ce qui indique qu'ils sont étroitement liés. La matière organique n'est en fait que la conséquence d'une transformation des glands en pâte. Ils sont donc le résultat d'un produit élaboré.

L'absence de cupule ou d'enveloppes qui entourent les glands montre qu'ils en ont été débarrassés avant d'être mis à cuire. Les glands donnés aux animaux ne nécessitent pas de transformation puisqu'ils les mangent tels quels. Cette préparation ne leur était donc certainement pas destinée mais plutôt vouée à l'alimentation humaine. De nombreux témoignages de consommation des glands par les hommes sont bien avérés, que ce soit chez les auteurs antiques ou encore dans les témoignages ethnographiques. Pline (XVI, 15) signale, par exemple, que certaines personnes, en cas de 


\begin{tabular}{|c|c|c|c|c|}
\hline Type de préparation & Type de cuisson & Forme des végétaux & Texture & Consistance \\
\hline "Grillage » & à sec & entiers & grains ou fruits secs & $\begin{array}{l}\text { restes entiers ou éventuellement } \\
\text { fragmentés }\end{array}$ \\
\hline Pain, galette & à sec & moulus (farine) & fine, régulière & épaisse, compacte \\
\hline \multirow[b]{2}{*}{ Bouillie } & \multirow[b]{2}{*}{ dans du liquide } & moulus (farine) & fine, régulière & assez épaisse \\
\hline & & entiers ou gruau & $\begin{array}{l}\text { plus ou moins grossière, fragments } \\
\text { de végétaux }\end{array}$ & assez épaisse \\
\hline Purée & dans du liquide & $\begin{array}{l}\text { entiers ou en morceaux, } \\
\text { écrasés ou moulus après } \\
\text { cuisson }\end{array}$ & $\begin{array}{l}\text { de grossière avec fragments de } \\
\text { végétaux à assez fine, régulière }\end{array}$ & épaisse \\
\hline Soupe & \multirow[t]{2}{*}{ dans du liquide } & entiers ou morceaux & $\begin{array}{l}\text { irrégulière, avec fragments de } \\
\text { végétaux }\end{array}$ & liquide \\
\hline Potage & & moulus & fine, régulière & liquide \\
\hline
\end{tabular}

Tableau 4 : consistance obtenue en fonction du type de préparation, du mode de cuisson et de la forme des végétaux utilisée. Table 4: consistency obtained depending on the type of preparation, cooking methods and form of plants used.

disette, réduisaient des glands séchés en farine qu'ils intégraient au pain lors de sa fabrication. Il évoque aussi en Espagne une cuisson sous la cendre des glands les plus doux qui sont mangés en dessert. Strabon $(3,3,7)$ parle aussi de pain de glands.

A. Maurizio (1932, p. 51-53) rapporte que les Indiens de Californie se nourrissaient, entre autre, de glands. Les coques des glands étaient brisées avec une pierre ronde et les cotylédons mis à sécher au soleil. Pour la mouture, on utilisait une pierre plate et une meule mobile allongée. La farine de glands était débarrassée de son amertume par un système de lessivage à l'eau chaude (l'eau passant à travers la farine emporte avec elle le tanin). Elle pouvait alors être utilisée à des fins alimentaires dans une cuisson à l'eau bouillante (soupe claire ou bouillie épaisse au goût peu prononcé rehaussé par des épices) ou aussi être rôtie, ou bien servir à l'élaboration de galettes avec une pâte de farine de glands. Les Tartares de Crimée, au début du siècle, faisaient dessécher les glands, puis en faisait une pâte avec de l'eau (Maurizio, 1932 : 92).

Plus proche de nous, la consommation de glands est actuellement attestée au Maroc. C'est le chêne liège qui est préférentiellement utilisé car ces fruits contiennent moins de tannins et sont donc plus doux. Ils sont mangés crus, grillés sur des braises, ou encore bouillis dans de l'eau puis séchés et réduits en farine ${ }^{8}$. La forme sous laquelle peut être consommée le gland est donc assez variée.

En ce qui concerne l'échantillon de Sublaines, la structure de l'agglomérat montre qu'il n'a pas subi de cuisson à sec de type grillage du fruit car les cotylédons sont en partie transformés en pâte. Il n'a pas non plus été élaboré à partir

8. [http://www.tela-botanica.org/page:chene]. de farine puisque des morceaux de cotylédons sont attestés. La cuisson dans un liquide est l'hypothèse la plus probable.

Selon la quantité de liquide, du temps de cuisson et du broyage final, ou non, des cotylédons, le résultat escompté correspondait à une soupe, une bouille ou une purée.

Ce genre de résidus n'est pas un cas isolé : à HauteriveChampréveyres à l'âge du Bronze, un fond de céramique évasée contenait une masse carbonisée uniforme avec des demi-glands décortiqués (Jacquat, 1989: 85).

\section{Les amas d'orge de Saint-Georges}

Comme il a été constaté plus haut, des grains d'orge sont visibles au sein des blocs carbonisés de matière organique. La préparation n’a donc pas été élaborée à partir de farine.

D'autre part, les grains sont agglomérés dans une pâte. Ils n’ont donc pas été grillés à sec mais cuits dans un liquide. La texture de ces agglomérats évoque des éléments assez grossièrement hachés, résultats probable de l'usage d'un gruau. Quelques grains entiers, de petites dimensions, ont cependant été repérés au sein du bloc carbonisé. Ces quelques grains ne suffisent pas à contredire l'hypothèse d'un gruau, leur présence pouvant s'expliquer de deux façons : une fois les grains concassés, ils ont pu être passés au crible afin de séparer le gruau des grains encore entiers. Les grains de petite taille ont alors pu passer à travers les mailles des cribles et être ainsi mêlés au gruau. Il est également envisageable que les grains d'orge aient préalablement été séparés selon leur taille à l'aide de cribles avant d'être concassés : les grosses semences auront été réservées à une utilisation autre tandis que les petites ont été broyées de façon grossière, laissant ainsi quelques grains sous forme entière dans le gruau. 
La texture des blocs de matière organique ainsi que la forme bombée régulière de ces amas, suggère une préparation culinaire qui a brûlé au cours de sa cuisson gardant ainsi la forme du récipient qui la contenait. Cela correspondrait à ce que C. Jacquat (1989) appelle " des croûtes de pots ", c'est-à-dire des restes de nourriture qui ont cuits dans une céramique et qui en ont conservés la forme incurvée. Ces amas ne sont donc pas un simple agglomérat de matériaux organiques, mais bien des déchets de préparation alimentaire.

D'après les observations portées sur les résidus carpologiques et les critères élaborés pour les divers types de préparations, les blocs peuvent être attribués à de la soupe ou de la bouillie. L'aspect compact évoque plutôt une bouillie mais ce phénomène peut n'être dû qu’à une évaporation massive de l'eau.

Un exemple de " croûte de pot » a été trouvé dans la Grotte des Planches incendiée à l'âge du Bronze final (Pétrequin et al., 1985). Cette " galette ", ainsi dénommée par les auteurs, a la forme d'un disque brisé de $10 \mathrm{~cm}$ de diamètre. Sa face supérieure est légèrement concave et très irrégulière, sa face inférieure est convexe. La carbonisation de ce produit s'est faite accidentellement lors d'un incendie, au fond d'une grande jarre biconique à col étroit. La structure interne de cet agglomérat est poreuse, vacuolaire; des fragments d'orge concassés et grossièrement écrasés sont visibles en surface. La forme de la jarre ainsi que l'absence de trace de chauffe dans sa partie inférieure incitent les auteurs à conclure qu'il ne s'agit pas d'une bouillie mais plutôt d'une technique de brassage de bière. Il nous semble que ce pourrait également être un gruau en cours de trempage pour amollir les grains.

Caton, auteur romain qui écrit vers 180 avant notre ère, décrit une recette de bouillie de froment : laver, écorcer et tamiser une demi-livre de froment pur. Le faire cuire dans une marmite avec de l'eau. Après la cuisson, on ajoute du lait jusqu’à former une crème épaisse (Caton, LXXXVI). On peut imaginer une recette de même type avec de l'orge.

\section{Le caramel alimentaire de Corbeilles}

La couche carbonisée du tesson de céramique de Corbeilles présente un aspect homogène, une texture fine sans aucune graine visible avec cependant quelques fragments de glumelles de millet commun. Plusieurs préparations peuvent être envisagées si l'on tient compte de la finesse de la texture : purée, soupe, bouillie ou encore pain/galette. Dans les cas de purée et de soupe, ce sont des végétaux entiers ou fragmentés qui sont mis à cuire. Qu'en est-il pour le millet découvert à Corbeilles : avant cuisson, était-il entier ou sous forme de gruau, décortiqué ou bien encore vêtu?
Les glumelles de millet, assez coriaces, sont réputées peu propices à la consommation. Si A. Maurizio (1932) mentionne que chez certaines peuplades, les bouillies et flans sont faits avec des graines écrasées mais non décortiquées (p. 295), les témoignages ethnologiques montrent un décorticage systématique des grains avant leur consommation (Toulemonde, dans ce numéro). Deux exemples archéologiques semblent cependant montrer le contraire.

Parmi les blocs carbonisés d'Hauterive-Champréveyres, datés du Bronze final, beaucoup sont constitués de grains de millet avec glumelles. C'est également le cas dans une céramique provenant d'un site suisse à proximité et daté de la même période, qui livre une croûte de $2 \mathrm{~cm}$ collée aux parois (Jacquat, 1989). Par ailleurs, plusieurs fonds de vases gallo-romains précoces dans la moyenne vallée de la Saône ont livré des résidus alimentaires carbonisés composés de millet commun possédant encore ses glumelles. L'hypothèse des auteurs est qu'il pourrait s'agir d'une préparation sous forme de bouillie ou de bière (Bonnamour \& Marinval, 1985). Concernant les vestiges de Corbeilles, l'hypothèse de bière n'est pas envisagée car cette boisson est élaborée à base de céréales germées trempées mais non broyées finement.

La pâte préservée dans la céramique de Corbeilles n'est pas régulière et aussi fine qu'elle devrait l'être dans le cas d'une préparation à base de farine. Cependant, les expérimentations de F. Toulemonde (dans ce numéro) montrent qu'une carbonisation de grains de millet entiers, avec ou sans eau, produit un résidu de grains agglomérés plus ou moins compact en fonction de la présence d'un liquide ou non. Ici, seules quelques glumelles sont visibles mais pas de grain. C'est donc une farine qui a servi à l'élaboration de cette préparation. La présence de quelques fragments de glumes indique que cette farine était constituée, si ce n'est en totalité, au moins en partie de millet. La texture broyée ne permet en effet pas d'affirmer avec certitude que le mélange était confectionné uniquement à partir de cette seule céréale. D'autre part, le nombre réduit de fragments de glumes permet d'envisager que les grains étaient décortiqués car, dans le cas contraire, les restes d'enveloppes seraient plus nombreux et l'aspect de la couche moins homogène.

Ce résidu pourrait provenir de la confection d'un pain ou galette ou encore d'une bouillie. Les pains/galettes sont à exclure car la couche carbonisée n'a pas l'aspect compact de ce type de préparation. Il s'agit donc d'un reste de bouillie confectionnée à partir de farine. Les exemples de consommation de bouillie de millet sont nombreux et A. Maurizio (1932) signale cette plante comme étant la céréale type pour les bouillies. 


\section{Le caramel alimentaire de Saran}

La texture fine et régulière de ce caramel évoque l'usage d'une farine. Cette préparation carbonisée peut être simple, à base de farine et de liquide qui permet d'obtenir une bouillie, ou bien plus complexe, constituée de farine à laquelle on a ajouté des œufs, du fromage frais, du miel... Dans ce cas, la seule observation visuelle sous loupe binoculaire ne permet pas d'avoir une idée de la composition du caramel.

La présence d'une préparation culinaire brûlée dans un vase dit de stockage peut sembler étrange. Il est cependant probable que les utilisations des céramiques n'étaient pas strictement limitées à un usage exclusif et que leur destination d'origine était ponctuellement détournée. On ne peut néanmoins exclure que la préparation carbonisée dans ce vase n'ait pas été destinée à l'alimentation. En effet, la fabrication de colle végétale à base de farine et d'eau que l'on fait chauffer ne forme pas autre chose... qu'une bouillie. Ce type d'adhésif, soluble à l'eau, est utilisé encore actuellement pour coller des matériaux légers tel le papier, le carton, des feuilles d'arbres ou encore du tissu.

\section{Conclusion}

Dans des contextes chronologiques proches, quatre exemples de préparations culinaires diversifiées, élaborées avec des ingrédients de base variés, ont pu être mis en évidence. Ils démontrent ainsi pour La Tène en région Centre, l'utilisation dans l'alimentation quotidienne de semences végétales sous forme entière, concassée et moulue pour la confection de bouillies et peut-être de soupes.

Les bouillies ont un avantage certain qu'elles ne nécessitent pas de préparation très élaborée contrairement au pain (farine et usage de levain, pétrissage, temps de repos...). Leur cuisson est simple, peut se faire partout aisément, tandis que celle du pain requiert l'usage d'un four. Elle ne nécessite pas de surveillance accrue puisque ce plat peut être mis à mijoter, d'où parfois quelques ratés de cuisson. Par ailleurs, toutes les espèces céréalières ne sont pas panifiables tandis que leur utilisation pour l'élaboration de bouillie est possible.

Outre les résidus de caramels alimentaires du type de ceux présentés ici, la découverte de fragments de matière organique carbonisée difficile à identifier est assez fréquente. Faut-il envisager des analyses systématiques sur ces résidus afin de les caractériser? Le coût d'une telle étude rend sa réalisation peu envisageable. Cette idée reste malgré tout alléchante même s'il est bien évident que nous ne connaî- trons jamais la recette exacte ni le tour de main du (de la) cuisinier(e).

\section{Remerciements}

Merci à Frédérique Durand, Marie-France Dietsch et Manon Cabanis, organisatrices de la Table ronde d'archéobotanique qui a eu lieu à Carcassonne en 2009, où fut présenté ce travail, et plus particulièrement à Frédérique Durand pour la relecture de cet article.

\section{Bibliographie}

Bonnamour, L. et Marinval, P., 1985. Céramiques galloromaines précoces avec dépôts de millet, dans la moyenne vallée de la Saône. RAE, XXXVI : 321-325.

Caton. Économie rurale.

Couderc, A. et al., 2006. Saint-Georges-sur-Cher « Le Marchais Rond " (Loir-et-Cher)/ Epeigné-les-Bois « Les Allets » (Indreet-Loire) : un établissement rural du second Âge du Fer (Tène $\mathrm{B}$ - Tène finale), Site $\mathrm{n}^{\circ} 46$, autoroute A.85, section M3. RFO, Inrap, SRA Orléans.

FrÉnÉE, E. (dir.) et al., 2008. A 85 Sublaines « Le Grand Ormeau », parcelle Cofiroute PP 47 à 49, 53, 54, 56 à 62 (Indre-et-Loire). $R F O$, Inrap, SRA Orléans.

Escallier, C. et Musset, D., 1991. Pétrir, frire, mijoter. Les cuisines des Alpes du Sud. Salagon, Les Alpes de Lumière, 108.

Horde, T., 2004. Dictionnaire des mots de la table. Sud-Ouest.

JACQUAT, C, 1989. Hauterive-Champréveyres, 2. Les plantes de l'âge du Bronze. Contribution à l'histoire de l'environnement et de l'alimentation. Saint-Blaise.

LABBÉ, M., 1997. Ces étonnants aliments végétaux fermentés et lactofermentés. Auvers-sur-Oise.

Lannoy, S., Marinval, P., Buléon, A., Chiron, H., Mejanelle, P., Pin, S., Rech, J., Tachpla, A., 2002. Études de "pains et galettes” archéologiques français. In K. Fechner, M. Mesnil (dir.). Pains, fours et foyers des temps passés, Civilisations, 49 (1-2) : 119-160.

Lusson, D. (dir.), 2009. Occupations rurales laténiennes et galloromaines à Saran "ZAC du Champ Rouge" (Loiret), sites 45.302.013 AH, tranches 2 et 32, zones 2 et 5. Rapport final d'opération de fouille archéologique. Inrap. SRA Orléans.

Maurizio, A., 1932. Histoire de l'alimentation végétale. Payot.

Pétrequin, P., Chaix, L., Pétrequin, A.-M., Piningre, F., La grotte des planches-près-Arbois (Jura). Proto-cortaillod et âge du Bronze final, 1985.

Pline l'Ancien. Histoire Naturelle. 
Poitevin, G. et al., 2007. Commune de Corbeilles (Loiret) "Franchambault». Site A19 K5. RFO. Inrap. SRA Orléans.

Pradat, B., 2001. Les écofacts : céréales et adventices. In O. Buchsenschutz, I. Ralston (dir.), L'occupation de l'âge du Fer dans la vallée de l'Auron à Bourges. Installations agricoles, funéraires et cultuelles ( $X^{e}-I^{e r}$ siècle avant J.-C.), collection Bituriga, Monographie 2001-2, supplément n 22 à la Revue Archéologique du Centre de la France, Bourges/Tours, 2001, 149-163.

Strabon. Géographie.

Toulemonde, F. (dans ce numéro). Les blocs carbonisés de millet commun de l'habitat protohistorique de Villiers-sur-Seine « Le
Gros Buisson » (77) : peut-on parler de préparation alimentaire?

Zech-Matterne, V., Bouby, L., Bouchette, A., Cabanis, M., Derreumaux, M., Durand, F., Marinval, P., Pradat, B., Sellami, M.-F. et Wiethold, J., 2009. L'agriculture du vi ${ }^{\mathrm{e}}$ au $\mathrm{I}^{\text {er }}$ siècle avant J.-C. en France : Etat des recherches carpologiques sur les établissements ruraux. In I. Bertrand, A. Duval, J. Gomez de Soto, P. Maguer (dir.). Habitats et paysages ruraux en Gaule et regards sur d'autres régions du monde celtique. Actes du XXXI ${ }^{\mathrm{e}}$ colloque international de l'Afeaf. Mémoire XXXV, $383-416$. 